\title{
Método Assistido por Computador e Prototipagem Rápida para o Fabrico Personalizado de uma Prótese de Dedo em Banda de Silicone
}

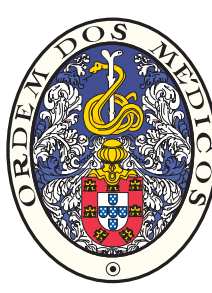

\section{Computer-Aided Method and Rapid Prototyping for the Personalized Fabrication of a Silicone Bandage Digital Prosthesis}

\author{
Nuno VENTURA FERREIRA ${ }^{1}$, Nuno LEAL ${ }^{2}$, Inês CORREIA SÁ ${ }^{1,3}$, Ana REIS ${ }^{2}$, Marisa MARQUES ${ }^{1,3}$
}

Acta Med Port 2014 Nov-Dec;27(6):775-779

\section{RESUMO}

O fabrico de próteses de dedos tem adquirido crescente importância não só pela recuperação psicossocial do doente mas também pela possibilidade de promover a prensão com pinça grossa. Apresenta-se um método de aplicação de tecnologias tridimensionais de desenho assistido por computador à produção de próteses passivas, através de um caso clínico de um doente com amputação do quinto dedo após reimplantação bilateral das mãos. Tomografia axial computorizada tridimensional foi utilizada para a elaboração de imagens antropométricas das mãos. Técnicas de desenho assistido por computador foram utilizadas para projectar a prótese digital a partir das imagens de reconstrução por inversão e superimposição do dedo contralateral. Recorreu-se ao método de manufactura por prototipagem rápida para a produção de um protótipo de prótese em banda de silicone. Esta abordagem vem substituir o método tradicional manual por um método virtual que estabelece um ponto de partida para a optimização de um inovador processo de elevada precisão e rapidez.

Palavras-chave: Cirurgia Plástica e Reconstrutiva; Dedos; Desenho Assistido por Computador; Próteses e Implantes; Silicones.

\section{ABSTRACT}

The fabrication of digital prostheses has acquired growing importance not only for the possibility for the patient to overcome psychosocial trauma but also to promote grip functionality. An application method of three dimensional-computer-aided design technologies for the production of passive prostheses is presented by means of a fifth finger amputee clinical case following bilateral hand replantation. Three-dimensional-computerized tomography was used for the collection of anthropometric images of the hands. Computer-aided design techniques were used to develop the digital file-based prosthesis from the reconstruction images by inversion and superimposing the contra-lateral finger images. The rapid prototyping manufacturing method was used for the production of a silicone bandage prosthesis prototype. This approach replaces the traditional manual method by a virtual method that is basis for the optimization of a high speed, accurate and innovative process.

Keywords: Reconstructive Surgical Procedures; Fingers; Computer-Aided Design; Prostheses and Implants; Silicones.

\section{INTRODUÇÃO}

O desenho e fabrico de próteses passivas de dedos requer uma complexa combinação de arte e ciência mas é sobretudo uma abordagem determinante na reabilitação psicossocial de doentes amputados. De facto, a amputação de dedos pode não só levar a afectação psicológica e social mas também económica do doente, dada a importância da mão e dos dedos na maioria das tarefas diárias. Considera-se hoje que a reabilitação protésica de doentes com segmentos amputados através de próteses passivas - tradicionalmente mal toleradas pelo doente e de difícil adaptação ao coto ${ }^{1}$ - vem sobretudo promover a sua integração psicossocial por meio de facilitar as actividades que impliquem prensão com pinça grossa e redução da hipersensibilidade do coto amputado.

Assim, a prótese deve idealmente replicar o dedo amputado tão precisamente quanto possível permitindo que o doente se exponha socialmente de forma discreta. Apesar de o fabrico de próteses de dedos ser actualmente um processo bastante satisfatório a nível estético, enquanto introduz um grau de estabilidade que permite à prótese funcionar também como suporte para o manuseamento de determinados objectos (movimentos grosseiros), a construção de próteses de dedos é ainda um processo sub-óptimo. Limitações relacionadas com a dificuldade e desconforto na retenção da prótese ${ }^{2}$ falta de realismo nos acabamentos finais e manutenção da coloração do material da prótese, ${ }^{3}$ e imprecisão do desenho e moldagem da prótese ${ }^{4}$ continuam ainda a ser objecto de optimização e pesquisa. Neste contexto, apresenta-se o caso clínico de um doente com amputação por necrose do quinto dedo da mão esquerda, 23 dias pós-reimplantação bilateral das mãos. Este doente foi proposto para o desenvolvimento de uma prótese passiva personalizada. Tendo como base as dimensões antropométricas do doente, um método digital e inovador para o desenho, projecção e fabrico de uma prótese passiva em banda para o segmento amputado foi desenvolvido por uma equipa interdisciplinar de médicos e engenheiros.

1. Departamento de Cirurgia. Faculdade de Medicina. Universidade do Porto. Porto. Portugal.

2. Instituto de Engenharia Mecânica e Gestão Industrial. Porto. Portugal.

3. Serviço de Cirurgia Plástica, Reconstrutiva e Estética. Hospital de São João. Porto. Portugal.

Recebido: 28 de Fevereiro de 2014 - Aceite: 18 de Agosto de 2014 | Copyright $\odot$ Ordem dos Médicos 2014 


\section{CASO CLÍNICO}

Sexo masculino, 21 anos, caucasiano, serralheiro, vítima de amputação traumática bilateral das mãos por esmagamento com quinadeira (Fig.s 1a-1c). Ao vigésimo terceiro dia pós-reimplantação bilateral das mãos, o quinto dedo da mão esquerda apresentou necrose estabelecida tendo sido cirurgicamente amputado (Fig. 1b). O doente é autónomo nas actividades diárias (Fig. $1 \mathrm{~d}$ ). ${ }^{5}$

Foi proposto para colocação de prótese passiva para o segmento amputado. Tradicionalmente as próteses passivas de dedos são fabricadas por um processo manual pouco preciso, que consiste sumariamente na produção de moldes negativos em gesso, alginato ou cera onde o(s) dedo(s) da mão simétrica são utilizados como referência, no vazamento do silicone (ou outro material) para o molde positivo, na coloração e acabamento final da prótese. Neste caso clínico foi desenvolvido um processo integrado digital de elevada precisão para o fabrico de uma prótese passiva em banda de silicone. $\mathrm{O}$ doente foi inicialmente submetido a uma tomografia axial computorizada com reconstrução tridimensional (TAC-3D) das duas mãos. Depois, os ficheiros DICOM (Digital Imaging and Communications in Medicine) contendo informação das imagens provenientes da TAC (Fig. 2a) foram segmentados em imagens de corte longitudinal e convertidos em ficheiros triangulados STL (stereo lithography) com auxílio do software MIMICS (Materialise $H Q$ ). (Fig. 2b).

De seguida, a zona de interesse para a concepção da prótese do quinto dedo foi seleccionada e modelada de acordo com medidas antropométricas contralaterais do doente com recurso a técnicas $3 \mathrm{D}$ de desenho assistido por computador ou 3D computer-aided design (CAD-3D) anatómico, tendo-se utilizado o software 3-Matic (Materialise $H Q$ ) (Fig. 2c). Tendo como base os ficheiros STL, foram impressos por prototipagem rápida SLA (estereolitografia) um macho para construção do molde em silicone (Smooth-on Inc.) (Fig.s 2d, 2e) e um macho de trabalho (Fig. 2f). Uma impressora de elevada precisão (Fig. 3a), a Viper $^{\text {TM }}$ SLA ${ }^{\circledR}$ System (3D Systems), foi utilizada para a prototipagem rápida dos modelos para fabrico da prótese final (Fig.s 3b, 3c). Os modelos prototipados foram curados em forno ultravioleta durante duas horas, o molde de silicone

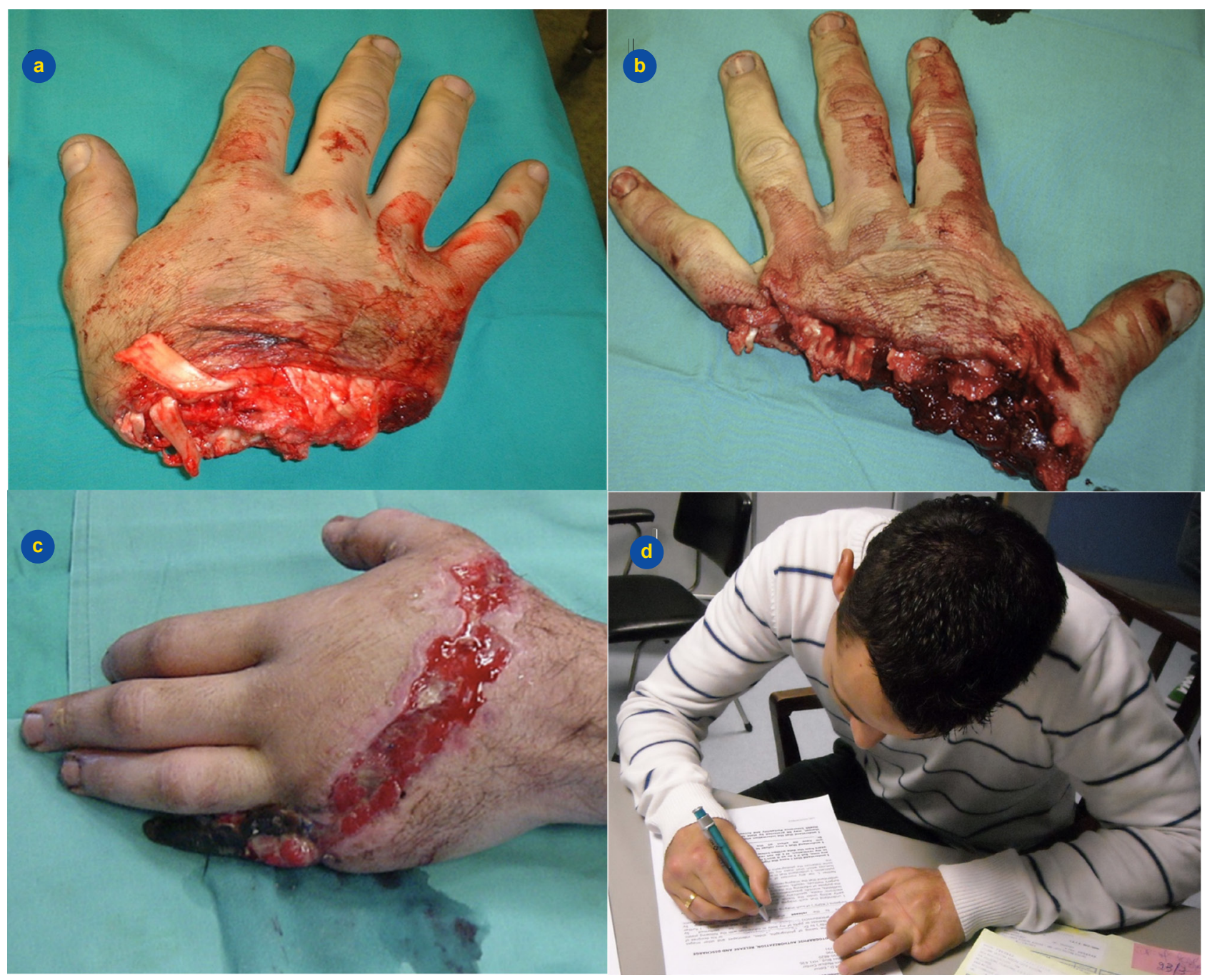

Figura 1 - Caso clínico. Amputação traumática bilateral das mãos - pré-operatório da mão direita (a) e da mão esquerda (b).Vigésimo terceiro dia pós-reimplantação bilateral das mãos: necrose estabelecida do quinto dedo da mão esquerda (c). Seguimento do doente cinco anos após cirurgia de reimplantação: escrita (d). 

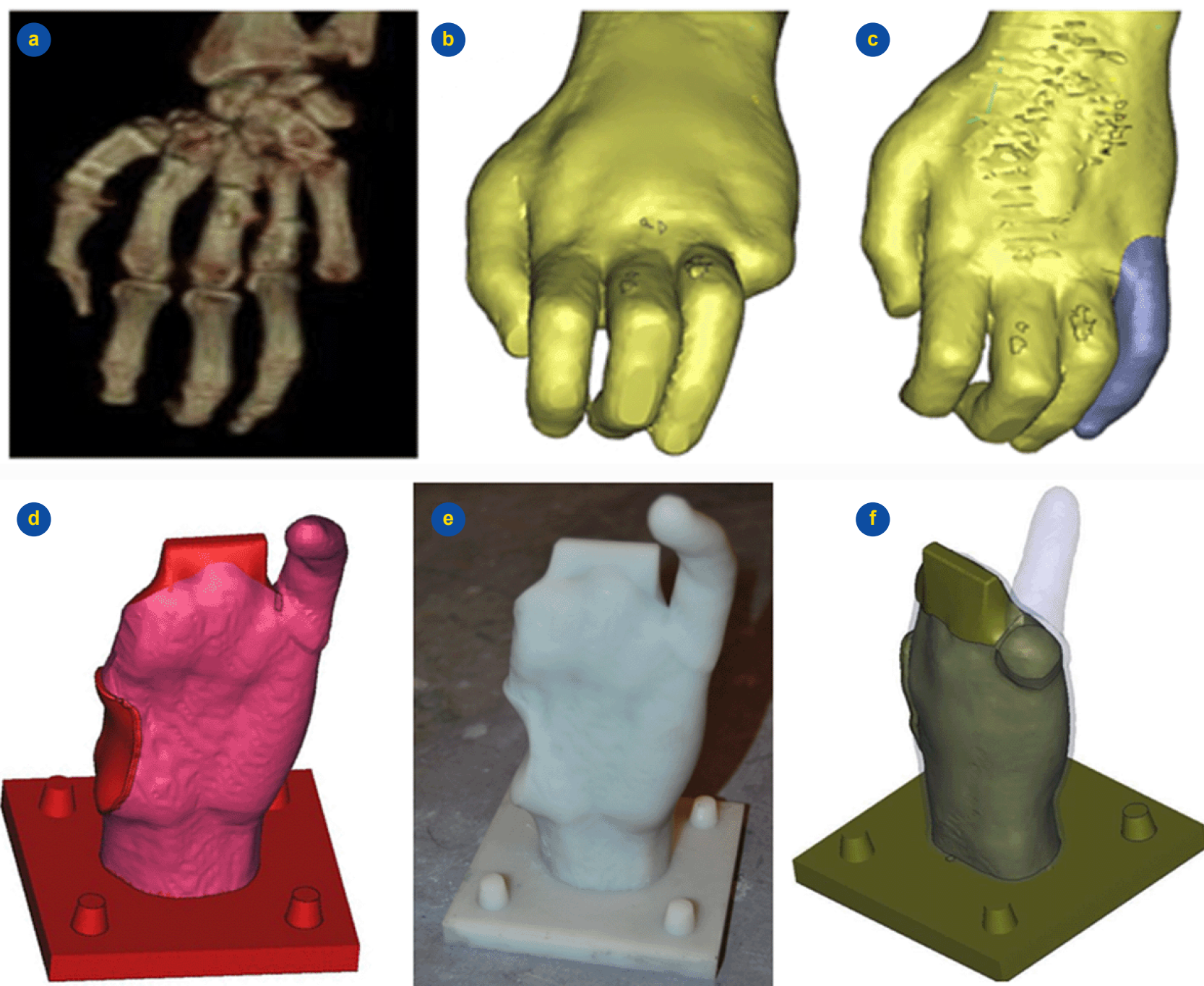

Figura 2 - Modelação da prótese e fabrico dos moldes. A partir da TAC-3D da mão do doente que foi segmentada em MIMICS (a), foi importado o biomodelo STL para 3-Matic (b). O plano sagital mediano das mãos do doente serviu como plano de simetria para a construção em 3-Matic da prótese (c), do macho para o molde de silicone (d, e) e do macho de trabalho para inserir no molde de silicone (f).

24 horas adicionais à temperatura ambiente. Na preparação do vazamento do silicone do protótipo (Fig.s 3d, 3e) foi usado desmoldante (HB Química), enquanto que a optimização da pigmentação do silicone foi obtida após experimentação de uma palete de cores (Siltone).

\section{DISCUSSÃO}

O nível de satisfação de doentes amputados que recebem prótese está directamente dependente do nível de personalização do seu processo de fabrico. O uso de 3D-CAD e prototipagem rápida na produção de próteses de dedos é aqui pela primeira vez validada em contexto clínico. A aplicação de programas como Solid Works (Dassault Systèmes SolidWorks Corp), Geomagic Studio (Raindrop Geomagic), FreeForm Modelling Plus (SensAble Technologies) ou 3-Matic surge hoje pontualmente reportada na literatura em contexto de investigação $0^{4,6}$ e de clínica, porém restrita ao fabrico de próteses maxilofaciais, ${ }^{7}$ auriculares, ${ }^{8}$ orbitais ${ }^{9} \mathrm{e}$ mamárias. ${ }^{10}$ Para além de substituir o tradicional processo de construção e fabrico manual de próteses de dedos este método virtual, integrado e digital de produção apresenta outras vantagens (Tabela 1). A elevada relação custo/benefício deverá diminuir assim que este e outros métodos semelhantes forem desenvolvidos para implementação clínica.

Relativamente à colocação de próteses do quinto dedo estas são as menos frequentes entre próteses de dedos, que são por si raramente apresentadas na literatura. A maioria dos estudos reportados tem-se focado exclusivamente no desenvolvimento ou aperfeiçoamento de técnicas de retenção ou acabamento. Os métodos mais frequentes para a retenção de próteses de dedo são a osteointegração, ${ }^{11}$ e a sucção por suspensão ${ }^{12}$ (criada pelo efeito de vácuo ao segmento residual ou coto) sem ou com auxílio de adesivos cutâneos, suportes mecânicos ou suportes anatómicos (Tabela 2). Dois métodos alternativos não-cirúrgicos para a retenção de próteses por sucção foram recentemente introduzidos por Leow et $\mathrm{al}^{13} \mathrm{e}$ por Arazpour et al. ${ }^{14}$ Estudos anteriores tinham já avaliado a aplicabilidade de próteses parciais em luva para substituição de múltiplos 

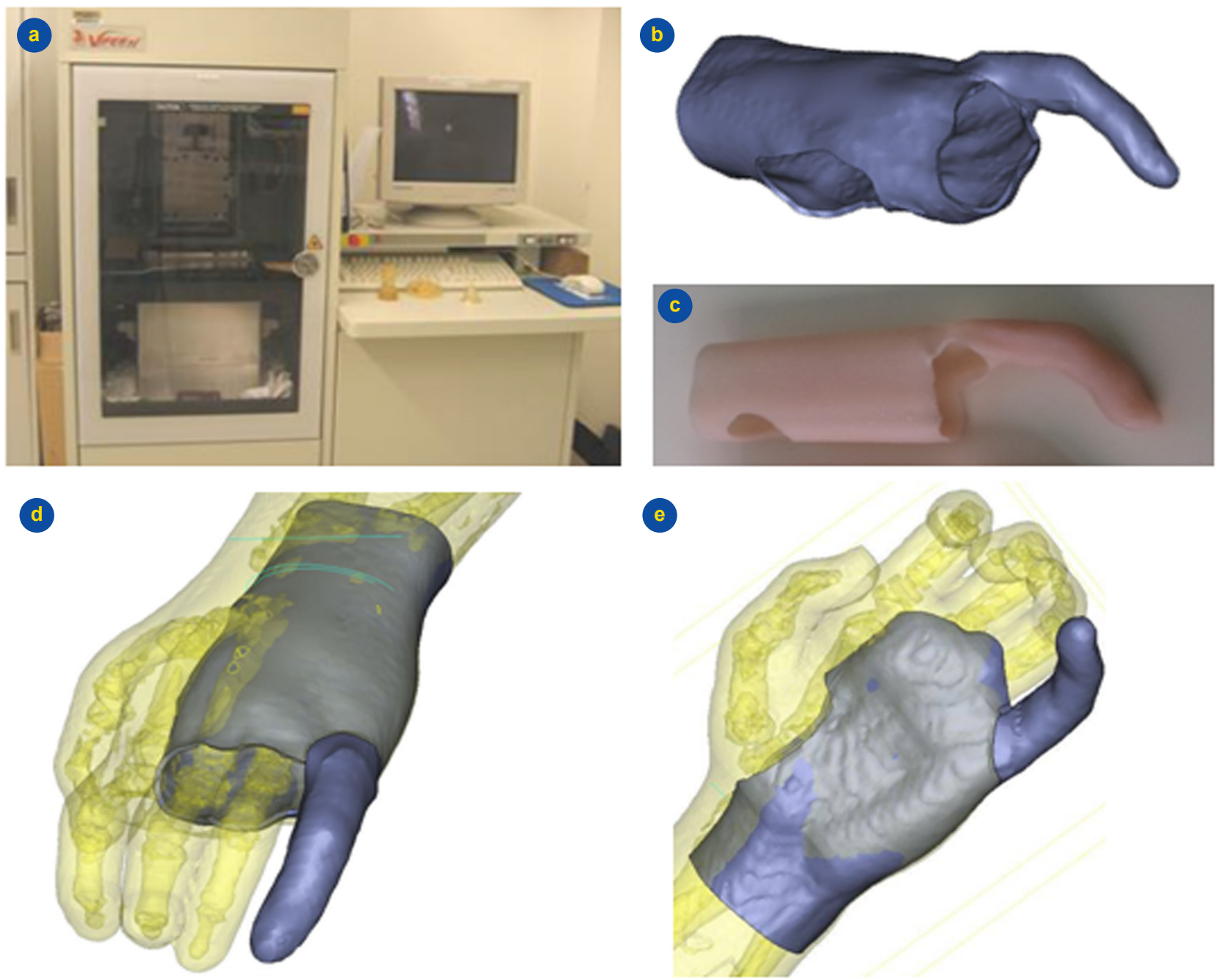

Figura 3 - Prototipagem rápida. Sistema SLA 3D de elevada precisão utilizada para a produção dos modelos para a prótese em estereolitografia (a). Projecção CAD-3D da prótese de dedo em banda (b) e prótotipo de prótese vazado em silicone (c). Imagem virtual da colocação da prótese, vista dorsal (d) e vista palmar (e) da mão.

Tabela 1 - Vantagens da técnica de fabrico de próteses passiva baseada em desenho auxiliado por computador e manufactura por prototipagem rápida

Vantagens

Rapidez na medição antropométrica

Rapidez no fabrico

Redução da quantidade de material usado

Precisão e perfeição

Personalização/Flexibilidade

Reproducibilidade

Redução do número de visitas do doente para planificação

dedos. ${ }^{15}$ Para além destes não são conhecidos outros métodos. O método de retenção aqui apresentado é baseado num design único em formato de luva que corresponde a uma alternativa viável e totalmente inovadora face aos métodos tradicionais de retenção.

Aqui apresenta-se um método inovador de colocação de prótese em banda de silicone desenvolvida após o doente, não candidato a colocação de prótese por sucção devido a ausência de coto do quinto dedo, ter sido contextualizado em 2007 da possibilidade de colocação de uma prótese osteointegrada com as seguintes implicações 1) necessidade de intervenção cirúrgica, 2) utilização obrigatória da prótese, 3) risco de infecção, e ter rejeitado essa solução. Com o desenvolvimento deste método a personalização e precisão da produção de uma prótese de dedo em banda foram maximizadas de forma a melhor corresponder às elevadas expectativas de um doente amputado numa era em que a Medicina busca cada vez mais soluções personalizadas.

\section{CONFLITOS DE INTERESSE}

Os autores declaram ausência de conflito de interesses.

\section{FONTES DE FINANCIAMEBNTO}

Este caso clínico está integrado no projecto estratégico PEst-OE/EME/LA0022 aprovado pela Fundação para a Ciência e Tecnologia no âmbito do programa de financiamento em 2013. 
Tabela 2 - Exemplos de relatos clínicos recentes reportando os dois métodos tradicionais e um método alternativo para o fabrico e colocação de próteses de dedos

\begin{tabular}{|c|c|}
\hline Autor & Relato clínico \\
\hline Doppen et al ${ }^{11}$ & $\begin{array}{l}\text { Reporta o resultado de três doentes com amputações ao nível da falange média ou distal num total de } 4 \text { dedos } \\
\text { que foram tratados com implantações osteointegráveis para colocação de próteses de dedos em silicone. É } \\
\text { reportada uma adaptação às técnicas tradicionais de osteointegração com o objectivo de reduzir o risco de } \\
\text { infeccão baseada nos principios de rotação regular do metal e limpeza com um agente de secagem após } \\
\text { colocação de brincos ou outros pierciengs corporais. Todos os doentes reportam satisfação cosmética e } \\
\text { funcional, inclusivé osteopercepção. Não são indicados detalhes de fabrico da prótese. }\end{array}$ \\
\hline Kamble et $\mathrm{al}^{12}$ & $\begin{array}{l}\text { Reporta a produção manual de próteses de dedos em dois casos clínicos de doentes com amputação de } \\
\text { múltiplos dedos. É detalhado o possesso de produção impressões em gesso odontológico, moldes em } \\
\text { cera, próteses finais em silicone e unhas em resina acrílica colorida. Para atenuar a linha de separação das } \\
\text { próteses aos dedos foram colocados anéis largos e/ou adesivos de cianoacrilato (super cola). As próteses } \\
\text { foram colocadas por sucção. Não ouve avaliação funcional ou de retenção da prótese. }\end{array}$ \\
\hline Leow et $\mathrm{al}^{13}$ & $\begin{array}{l}\text { Avalia um método alternativo de retenção de próteses baseado na colocação de um tubo de Micropore }{ }^{\mathrm{TM}} \text {-PVC } \\
\text { para a extensão do comprimento de segmentos residuais de dedos amputados menores do que os } 15 \text { mm } \\
\text { necessários para retenção de próteses de silicone. O estudo compara directamente este novo método com o } \\
\text { método de colocação de prótese por sucção com e sem adesivo cutâneo num total de } 10 \text { doentes. O nível de } \\
\text { retenção das próteses foi quantificado de acordo com a avaliação dos doentes. Não são indicados detalhes } \\
\text { de fabrico da prótese. }\end{array}$ \\
\hline
\end{tabular}

\section{REFERÊNCIAS}

1. Goldner RD. Replantation. In: Wolfe SW, Pederson WC, Kozin SH, editors. Green's Operative Hand Surgery. $6^{\text {th }}$ ed. Philadelphia: Elsevier Churchill Livingstone; 2011. p. 1585-601.

2. Aydin C, Karakoca S, Yilmaz H. Implant-retained digital prostheses with custom-designed attachments: a clinical report. J Prosthet Dent. 2007;97:191-5.

3. Tanner P, Leachman S, Boucher K, Ozcelik TB. Depigmented skin and phantom color measurements for realistic prostheses. Skin Res Technol. 2014;20:37-42.

4. Colombo G, Facoetti G, Rizzi C. A digital patient for computer-aided prosthesis design. Interface Focus. 2013;3:20120082.

5. Marques M, Correia-Sa I, Festas MJ, Silva S, Silva Al, Silva A, et al. Six years of follow-up after bilateral hand replantation. Chir Main. 2013;32:226-34.

6. Wang P, Hu J, Ma PX. The engineering of patient-specific, anatomically shaped, digits. Biomaterials. 2009;30:2735-40.

7. Verdonck HW, Poukens J, Overveld HV, Riediger D. Computer-assisted maxillofacial prosthodontics: a new treatment protocol. Int J Prosthodont. 2003;16:326-8.

8. Eggbeer D, Bibb R, Evans P. Toward identifying specification requirements for digital bone-anchored prosthesis design incorporating sub- structure fabrication: a pilot study. Int J Prosthodont. 2006;19:258-63.

9. Wu G, Bi Y, Zhou B, Zemnick C, Han Y, Kong L, et al. Computer-aided design and rapid manufacture of an orbital prosthesis. Int J Prosthodont. 2009;22:293-5

10. Eggbeer D, Evans P. Computer-aided methods in bespoke breast prosthesis design and fabrication. Proc Inst Mech Eng H. 2011;225:94-9.

11. Doppen P, Solomons M, Kritzinger S. Osseointegrated finger prostheses. J Hand Surg Eur Vol. 2009;34:29-34.

12. Kamble V, Desai R, Arabbi K, Mahajan k, Patil S. Fingers prosthesis for multiple finger amputations: two case reports. National Journal of Medical and Dental Research. 2013;1:38-42.

13. Leow $M$, Chong A, Peng $Y$, Pho R. Fitting very short finger stumps with silicone prosthesis: a nonsurgical method. Prosthet Orthot Int. 2013;37:415-20.

14. Arazpour M, Mardani MA, Ahmadi Bani M, Zarezadeh F, Hutchins SW. Design and fabrication of a finger prosthesis based on a new method of suspension. Prosthet Orthot Int. 2013;37:332-5.

15. Leow ME, Kour AK, Ng WK, Pho RW. Creating a model for fabricating a partial hand glove prosthesis using the realigned casts of the contralateral digits. Prosthet Orthot Int. 1999;23:72-4. 


\section{Método Assistido por Computador e Prototipagem Rápida para o Fabrico Personalizado de uma Prótese de Dedo em Banda de Silicone}

Acta Med Port 2014:27:775-779

Publicado pela Acta Médica Portuguesa, a Revista Científica da Ordem dos Médicos

Av. Almirante Gago Coutinho, 151

1749-084 Lisboa, Portugal.

Tel: +351218428215

E-mail: submissao@actamedicaportuguesa.com

www.actamedicaportuguesa.com

ISSN:0870-399X | e-ISSN: 1646-0758

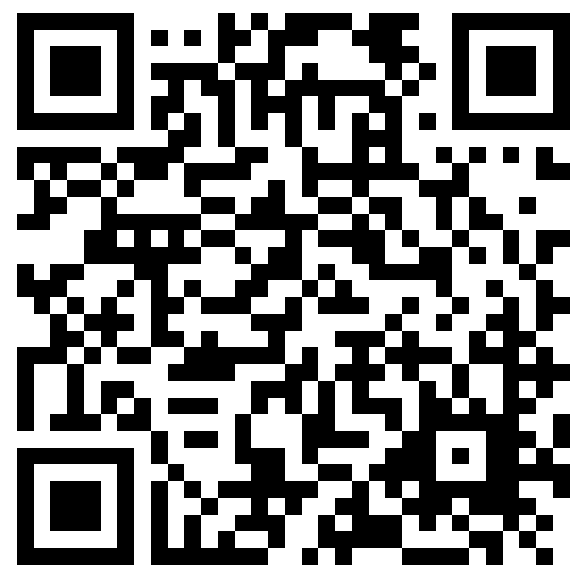

